\title{
HARDY-BENNETT-TYPE THEOREMS
}

\author{
L. LEINDLER
}

Abstract. The factorization of inequalities, introduced and treated systematically by G. Bennett, is a new and very effective method providing the best possible version of several classical and recent inequalities. Here we moderately improve two factorization-theorems proved by us.

Mathematics subject classification (1991): 26D15, 40A05, 40A99.

Key words and phrases: Inequalities for sums, factorization, $l^{p}$-spaces, classical and recent inequalities.

\section{REFERENCES}

[1] G. BEnNeTt, Some elementary inequalities, Quart. J. Math. Oxford 38 (1987), 401-425.

[2] G. BenNeTt, Factorizing the Classical Inequalities, Memoirs of Amer. Math. Soc. 120 (1996), Number $576,1-130$

[3] L. LEINDLER, A theorem of Hardy-Bennett-type, Acta Math. Hung. 78(4) (1998), 315-325.

[4] L. LeINDLER, Two theorems of Hardy-Bennett-type, Acta Math. Hung. 79(4) (1998), 341-350. 